\title{
Tú y Usted en la ciudad de México. ¿Qué tanto y cómo influyen el sexo, la edad y el nivel educativo?*
}

\author{
Tú and Usted in Mexico City. How much and how does gender, age, and \\ instruction affect them?
}

\author{
Cristal Yeseidy Cepeda Ruiz
}

\begin{abstract}
Resumen
En este estudio nos centramos en los tratamientos de segunda persona singular tú y usted usados en el español de la Ciudad de México, en relación con tres variables sociales sexo, edad y nivel educativo (del hablante y del oyente). A partir de la frecuencia y distribución de las formas pronominales, así como de las valoraciones cualitativas de los encuestados recreamos el sistema de tratamientos actual y lo comparamos con información de investigaciones previas. Los datos indican que la edad, el nivel educativo y el sexo de los encuestados y de sus (inter)locutores son relevantes en la elección de una u otra forma pronominal; el cotejo con estudios anteriores permite apreciar un ligero aumento de $t u ́$ frente a usted desde la década de los setenta, así como evidenciar un patrón de estratificación por edad caracterizado por el uso de tú cuando los hablantes son jóvenes y usted cuando los colaboradores llegan a la adultez. Los resultados cuantitativos y cualitativos que ofrecemos provienen de 48 cuestionarios sociolingǘsticos aplicados a capitalinos y migrantes internos que residen en la capital mexicana, hombres y mujeres de cuatro generaciones y tres niveles educativos.
\end{abstract}

\section{Palabras clave}

formas de tratamiento, estratificación por edad, tiempo aparente, tiempo real

\begin{abstract}
In this study we focus on the second person singular pronouns tú and usted used in the Spanish of Mexico City, based on three social variables gender, age and educational level (of the speaker and the listener). Throughout the frequency and distribution of these pronominal forms, as well as according to the qualitative perception and the collaborators' evaluation, we explore the current system of personal treatment and compare it with data obtained in previous studies. Our data indicates that the age, the educational level and the gender of the speakers are relevant in the choice of one or another pronominal form. The comparison with previous studies allows us to appreciate a slight increase of tú in front of usted as from the Seventies; it also allows us to show an age-graded pattern characterized by the use of tú when the speakers are young, and usted when they reach the adulthood. Quantitative and qualitative data come from 48 sociolinguistic questionnaires distributed among speakers from Mexico City as well as internal migrants residing in the Mexican capital, men and women of four generations and three different educational levels.
\end{abstract}

\section{Key words}

Forms of address, age grading, apparent time, real time.

\footnotetext{
* Este estudio hace parte de la investigación de doctorado 'Formas pronominales y fórmulas nominales de tratamiento en el español de la Ciudad de México" (Cepeda: en curso), estudio financiado por el Consejo Nacional de Ciencia y Tecnología de México (CONACyT). Agradezco a los doctores María Ángeles Soler Arechalde, Pedro Martín Butragueño y Julio Serrano Morales por la asesoría brindada durante el transcurso de esta investigación; los errores, indudablemente, son todos míos. Igualmente, agradezco a todos aquellos que participaron en este estudio, ya como enlaces para conformar la muestra, ya como encuestados.
} 
Cristal Yeseidy Cepeda Ruiz, Universidad Nacional Autónoma de México, cristalcepedaruiz@,comunidad.unam.mx

Recibido: junio 2018 / Aceptado: octubre 2018

DOI 10.17710/tep.2018.4.1.1garciaramon

\section{Introducción}

Se conocen como formas de tratamiento los sistemas pronominales de segunda persona del singular — tú, usted, vos, sumercé — 1 y del plural —ustedes, vosotros — que usa el hablante al dirigirse directamente a su(s) oyente(s) (Carricaburo, 1997; RAE-ASALE, 2009; Fontanella de Weinberg, 1999; Hummel, Kluge y Vázquez, 2010; Bertolotti, 2015; entre otros); dichos tratamientos se manifiestan en la lengua mediante tres estrategias: “[...] toda forma de tratamiento puede descomponerse en tres partes: elementos nominales, formas pronominales y formas verbales [...]" (Calderón, 2010, p. 553). ${ }^{2}$

Hablar del sistema de tratamientos pronominales usado por una comunidad consiste, sin duda, en retratar el entorno social de los sujetos que la conforman; estudiar el tratamiento de un grupo implica analizar la relación que existe entre el trato pronominal y factores sociales del locutor y de su posible interlocutor como la edad, clase social, el sexo, nivel educativo, entre otros (Hummel, Kluge y Vázquez, 2010 p. 15).

Según la forma pronominal singular que predomine en una región, el mundo hispanohablante ha sido clasificado en tres grupos: territorios tuteantes, voseantes y ustedeantes (Carricaburo, 1997; Fontanella de Weinberg, 1999 y Bertolotti, 2015). ${ }^{3}$ En consecuencia, Carricaburo (1997) y Fontanella de Weinberg (1999) agrupan a México dentro de los países predominantemente tuteantes: "Es netamente tuteante la mayor parte del territorio mejicano [...]" (Carricaburo, 1997, p. 19); adicionalmente, la literatura reporta para la República mexicana el uso de usted en contextos formales y de distancia, en tanto que tú se destina a situaciones íntimas y de confianza: “[...] es propio del español de México el

\footnotetext{
${ }^{1}$ Para más información sobre el carácter pronominal de sumercé véase Cepeda (2014) y Bertolotti (2015).

$2 \mathrm{Al}$ respecto, Bertolotti (2015, p. 10) indica que: "El tratamiento alocutivo en la lengua española se expresa gramaticalmente (formas pronominales — personales y posesivos — o verbales) y nominalmente".

3 Una de las clasificaciones más relevantes para el español es la que propone Fontanella de Weinberg (1999), autora que considera la existencia de cuatro sistemas pronominales, según se utilicen las formas singulares tú/vos/usted y plurales vosotros/ustedes en los ámbitos formal/confianza/intimidad. Una propuesta más reciente es la de Bertolotti (2015, p. 71) quien añade un nuevo sistema de trato en el que se incluye la forma sumercé, esto con el fin de dar cuenta de los usos de algunas regiones de países como Colombia, Ecuador, Perú y República Dominicana.
} 
sistema de tratamiento que opone los pronombres singulares tú y usted, el primero para el trato de confianza y el segundo, para el trato deferente y de distancia” (Vázquez y Orozco, 2010, p. 249).

Así mismo ocurre con la Ciudad de México, este territorio suele considerarse tuteante, según lo indican los hablantes (especialmente los foráneos que no residen en la ciudad) y algunos de los pocos trabajos que analizan el tratamiento exclusivamente en la capital mexicana (Lastra, 1972). Esta idea, sin embargo, debe tomarse con mucha precaución; por un lado, las investigaciones de corte sociolingüístico más completas no son recientes (Lastra, 1972 y Kim Lee, 1989); por otro lado, la información actual se concentra en tan solo un sector de la población (la clase media, por ejemplo) o incluye a sujetos de varias regiones bajo la etiqueta 'mexicanos' (Schwenter, 1993; Reid y Comajoan; Pejušković, 2013 y Rojas, 2014). En síntesis, la información de la que disponemos no nos permite confirmar el tuteo como forma pronominal no marcada de la Ciudad de México; no sabemos, por ejemplo, si su uso es generalizado o sectorizado; desconocemos quiénes (y en qué situaciones) utilizan tú y quiénes usted en la actualidad.

En estas líneas examinaremos el sistema pronominal de tratamientos usado por los colaboradores — trato dirigido-, así como el esperado de sus (inter)locutores en contextos bien delimitados — trato recibido-; ${ }^{4}$ observaremos si tú es la forma más frecuente en la actualidad, si su uso es generalizado o si, por el contrario, existen situaciones en las que usted es más reportado por los hablantes. La investigación, además, se centra en determinar la relación entre los factores extralingüísticos $\longrightarrow$ sexo, edad, nivel educativo- en la selección de tú y usted en la Ciudad de México, así como las diferencias o similitudes entre dichos factores en el pasado y en la actualidad.

Nuestro estudio busca reflejar, si no estadísticamente, de manera cualitativa, la gran diversidad de la capital mexicana; por lo mismo, incluimos a 48 hablantes, unos que han nacido en la Ciudad de México y otros que han migrado de otras regiones para habitar la metrópoli, 21 hombres y 27 mujeres desde los 11 hasta los 69 años, con diferentes profesiones, oficios y grados de escolaridad, monolingües en español (42) y bilingües con dominio en una lengua indígena (6): matlazinca, náhuatl, mixteco y zapoteco.

La hipótesis que defendemos en estas líneas señala una fuerte relación entre los factores sociales edad, sexo y nivel educativo tanto del emisor como del receptor en la

\footnotetext{
${ }^{4}$ Given/ received, según Jaramillo (1990, p. 16). 
selección/recepción de las formas pronominales/verbales de tratamiento en el español de la Ciudad de México; más allá, en este estudio reconocemos sectores de la población y contextos situacionales en los que usted prevalece como trato ante tú y cuestionamos, a partir de los resultados actuales, la idea que considera que el pronombre tú es de uso generalizado en la Ciudad.

El documento se organiza como sigue. En la primera sección —introducciónpresentamos el problema de estudio, la justificación, los objetivos y la hipótesis de esta investigación. Seguido de esto, en los antecedentes, proporcionamos al lector información sobre la Teoría del Poder y la Solidaridad de Brown y Gilman (1960), encontramos una descripción del sistema de tratamientos reportado para la Ciudad de México en investigaciones previas, nos enfocamos en la relación entre tú/usted y las variables sociales analizadas. Posteriormente, se ubica la metodología de este estudio; detallamos información sobre la muestra, las variables de análisis y el instrumento utilizado para recoger datos. Seguido de esto, el lector encontrará los resultados cuantitativos y cualitativos obtenidos en este trabajo, nos centramos en la distribución y frecuencia actual de los tratamientos a partir de las variables sociales y cotejamos dichos resultados con los obtenidos en investigaciones anteriores. Finalmente, señalamos las conclusiones del presente trabajo y ofrecemos la bibliografía básica.

\section{Antecedentes}

\subsection{Generalidades sobre el trato}

La Nueva Gramática de la Lengua española define las formas de tratamiento como “[...] las variantes pronominales que se eligen para dirigirse a alguien en función de la relación social que existe entre el emisor y el receptor (tú, usted, vos, os, le, te, etc.)" (RAE-ASALE, 2009, p. 1250). En el caso particular del español, debido a que se trata de una lengua prodrop, ${ }^{5}$ el tratamiento puede codificarse de manera explícita, es decir, con la mención directa del pronombre (I) o de manera tácita, mediante la concordancia con el verbo y con el paradigma flexivo 6 (II):

\footnotetext{
5 Entiéndase por lengua pro-drop (prodrop) aquella en la que el sujeto de una cláusula puede ser elidido sin afectar esto la gramaticalidad de la misma: "A term used in government-binding theory for a parameter which determines whether the subject of a clause can be suppressed. [...] Other properties of pro-drop languages have been suggested, such as that they have a rich system of verb-agreement, and free inversion of subject and verb. Pro-drop languages are also known as 'null subject' languages" (Crystal, 2008, p. 389).

${ }^{6}$ Sujeto: tú, usted, ustedes; objeto: te, lo/la/le, los/las/les; reflexivo: te, se, se; término de preposición: ti/contigo, usted, ustedes; posesivo: tu/s tuyo/a/os/as, su/s suyo/a/os/as, su/s suyo/a/os/as, de ustedes.
} 
I. $\underline{\text { Usted }}$ tiene todo para ser feliz.

II. ¿Ø tienes tu carro en la puerta?

Los tratamientos pronominales pueden ser reciprocos/simétricos cuando los dos participantes del evento comunicativo usan la misma forma $-t \dot{u} \leftrightarrow \rightarrow$ tú, usted $\leftrightarrow \rightarrow$ usted y sus correspondientes plurales-, en tanto que se habla de tratamientos no recíprocos/asimétricos en casos en los que los sujetos utilizan diferentes pautas pronominales para tratarse —usted $\leftarrow \rightarrow$ tú. Adicionalmente, los tratamientos pronominales se clasifican en inestables/variables cuando el hablante selecciona el trato a partir de sus rasgos sociales y los de su interlocutor, así como de factores discursivos y pragmáticos; por su parte, se habla de tratamientos estables/invariables cuando el individuo no repara en dichos factores para elegir la forma pronominal/verbal y, por lo tanto, usa el mismo trato en todas sus interacciones comunicativas (RAE-ASALE, 2009, p. 1250).

Siguiendo la tradición, a los pronombres de tratamiento se les suelen asignar valores dicotómicos formal/informal, confianza/respeto, cercanía/lejanía, familiar/cortés (Brown y Gilman, 1960; Carricaburo, 1997; Fontanella de Weinberg, 1999); así, en español solemos asociar al tuteo con contextos de confianza, cercanía, intimidad o familiaridad, en tanto que es común definir el ustedeo como un trato prototípico para codificar respeto, lejanía o formalidad entre el hablante y oyente. No obstante, los estudios recientes reconocen la polivalencia de los tratamientos, su capacidad para codificar múltiples valores dependiendo del contexto comunicativo y de la comunidad de habla (Montes Giraldo, 1985; Oliveira, 2010; Bertolotti, 2015; entre otros):

[...] estas dicotomías no reflejan la situación de varios estándares hispanoamericanos. Por un lado, varios de ellos tienen sistemas tríadicos. Por otro lado, las mismas formas pueden ocupar más de una casilla, en aparentes contradicciones. Tal es el caso de hispanohablantes que usan la forma usted tanto para la intimidad como para la formalidad. [...] Estos pares de términos terminan no resultando satisfactorios porque resultan recoger un rasgo y dejan caer otros (Bertolotti, 2015, pp. 13-14, 20).

La solución a este inconveniente puede ser, uno, utilizar todos estos valores y seleccionar el que más se adecue al contexto particular (Carricaburo, 1997, p. 10); y dos, proponer una terminología mucho más general que abarque el contenido multifacético de los tratamientos (Bertolotti, 2015, p. 21). 


\subsection{La teoría del poder y la solidaridad (Brown y Gilman, 1960)}

Tal vez el trabajo más representativo sobre las formas de tratamiento pertenece a Roger Brown y Albert Gilman. Su investigación establece las bases teóricas y metodológicas para innumerables investigaciones sobre formas pronominales y aporta invaluable información tipológica sobre el sistema de trato en el español, francés, inglés, alemán e italiano. La teoría de Brown y Gilman posee dos conceptos fundamentales para entender el trato: la semántica ${ }^{7}$ del PODER y de la SOLIDARIDAD:

El supuesto es que en toda sociedad humana existe alguna clase de concepción acerca del valor social diferencial y acerca de la solidaridad diferencial, y que este par de dimensiones que rige la mayor parte de la vida social también rige los usos de tratamiento pronominal diádico (Wainerman, 1976, p. 48)

Es decir, la teoría considera que las relaciones interpersonales involucran estas dos dimensiones semánticas (PODER y SOLIDARIDAD) y que, como consecuencia, esto se refleja en las elecciones pronominales de los sujetos. El PODER es definido por Brown y Gilman como la capacidad que tiene un individuo de influir en el comportamiento de otro, involucra a un agente que actúa volitivamente y a un paciente que responde conforme a las motivaciones del primero; este tipo de relación se basa generalmente en las diferencias entre los individuos (edad, clase social, sexo, cargo en una institución, etc.), el PODER se relaciona con el eje vertical de las relaciones sociales, se jerarquiza a los individuos y se favorece el trato no recíproco: el superior trata familiarmente a un inferior $(T),{ }^{8}$ en tanto que este último usa una forma cortés al dirigirse a su superior $(V)$ :

One person may be said to have power over another in the degree that he is able to control the behavior of the other. Power is a relationship between at least two persons and it is nonreciprocal in the sense that both cannot have power in the same area of behavior. The power semantic is similarly nonreciprocal, the superior says $\mathrm{T}$ and receives $\mathrm{V}$. There are many basis of power -physical strength, wealth, age, sex, institutionalized role in the church, the state, the army or within the family (Brown y Gilman, 1960, p. 255).

Por su parte, la SOLIDARIDAD prioriza la igualdad y los sujetos se aprecian como miembros de un mismo grupo (familiar, religioso, político, de edad, sexo, etc.), por lo tanto, se concibe como el eje horizontal de las relaciones sociales. La SOLIDARIDAD promueve el trato recíproco y puede generarse y afianzarse a partir de características que son

\footnotetext{
7 "El término semántico se refiere aquí a la covariación entre el pronombre usado, y, por otro lado, el estatus objetivo y la relación de solidaridad existentes entre el hablante y su interlocutor" (Wainerman, 1976 p. 48).

${ }^{8}$ Las iniciales $V$ y $T$ son usadas ampliamente en la bibliografía sobre formas de tratamiento; dicha terminología es acuñada por los autores a partir de los tratos $t u \mathrm{y}$ vos provenientes del latín: "As a convenience we propose to use the symbols $T$ and $V$ (from Latin $t u$ and vos) as generic designators for a familiar and polite pronoun in any language" (Brown \& Gilman, 1960, p. 254).
} 
apreciadas por los individuos como afines en su manera de pensar y de 'actuar', así como del contacto frecuente entre los individuos (aunque no todos los encuentros generan hechos compartidos entre $A$ y $B$ ):

Now we are concerned with a new set of relations which are symmetrical; for example, attended the same school or have the same parents or practice the same profession, If $A$ has the same parents as $B, B$ has the same parents as $A$. Solidarity is the name we give to the general relationship and solidarity is symmetrical. The corresponding norms of address are symmetrical or reciprocal with $V$ becoming more probable as solidarity declines (Brown y Gilman, 1960, p. 258).

No todas las diferencias entre individuos pueden ser definidas en términos de PODER, ni todas las semejanzas promueven la SOLIDARIDAD; como señalamos arriba, estas dimensiones semánticas se vinculan directamente con las particularidades de una comunidad (las sociedades evalúan de manera diferencial factores como el sexo, el color de piel, la pertenencia a una casta, el peso de los sujetos, etc.):

Las diferencias vinculadas con el poder social dan origen a la aparición de $V$ en una dirección del tratamiento; las no vinculadas con el poder social dan origen a la aparición de $V$ en ambas direcciones; y las similitudes que hacen la solidaridad dan origen a la aparición de $T$ en ambas direcciones. (Wainerman, 1976, p. 49). ${ }^{9}$

Varias son las críticas que recibe el modelo teórico de Brown y Gilman. Tal vez las más interesantes son las relacionadas con el carácter dicotómico y la implementación de los conceptos familiaridad, confianza, respeto y formalidad (Oliveira, 2010, p. 60; Bertolotti, 2015, pp. 13-14, 21; entre otros); primero, por la definición no unívoca de dichos términos; segundo, por el carácter generalizador que vincula un único valor semántico a un pronombre de tratamiento, con lo cual se omite que: "los hablantes sustituyen las interpretaciones convencionales de las formas con otras nuevas a la hora de negociar un patrón de tratamiento no convencional según las normas sociales" (Oliveira, 2010, p. 60), como en el caso del tuteo de enojo usado en la Ciudad de México; y tercero, por ignorar los múltiples matices de las relaciones sociales y, por lo tanto, de los sistemas de tratamiento

\footnotetext{
${ }^{9}$ Wainerman retoma el estudio de Brown (1965) para definir los tipos de relaciones que se establecen entre $A$ y $B:[\ldots]$ Dos grupos importantes están constituidos por relaciones simétricas: cuando $A$ y $B$ son similares, cada uno tiende a gustar del otro, busca estar en compañía del otro, y cada uno se dirige con $T$ al otro. El conjunto opuesto de relaciones simétricas es como sigue: cuando $A$ es distinto de $B$, cada uno tiende a no gustar del otro, a no estar en compañía del otro, y cada uno dice $V$. Otros dos grupos importantes están constituidos por relaciones asimétricas: cuando $A$ tiene un valor social mayor que $B, A$ se siente superior y $B$ inferior [...] $A$ dice $T$ y $B$ dice $V$. El conjunto opuesto está constituido por las mismas relaciones pero con los roles de $A$ y $B$ invertidos. (Brown, 1965, p. 73; citado por Wainerman, 1976, p. 50).
} 
8 - Cepeda Ruiz, C. Y.

(Calderón, 2010; Oliveira, 2010 y Bertolotti, 2015), situación apreciable en el sumercedeo colombiano que involucra un valor intermedio o T/V (Cepeda, 2014).

\subsection{El sistema pronominal de la Ciudad de México}

En este apartado, basados en la documentación recopilada por Vázquez y Orozco (2010) y otros materiales, señalaremos algunos aspectos relevantes sobre las investigaciones (con énfasis en las de corte sociolingüístico) que estudian el tratamiento pronominal de la capital mexicana.

Vázquez y Orozco (2010) recopilan una serie de investigaciones —dialectológicas, diacrónicas, situacionales, documentales y sociolingüísticas - que dan cuenta del uso de las formas de tratamiento en diferentes lugares de la República mexicana; de estas investigaciones, las que se remiten específicamente al trato en la Ciudad de México son escasas (tabla 1) y abordan el tema, en algunos casos, de manera parcial.

\section{Tabla 1}

Estudios sobre formas de tratamiento en la Ciudad de México, adaptado de Várquezy Orozco (2010, p. 262)

\begin{tabular}{|l|l|}
\hline Dialectológicos & Guerrero Rubín (1985) \\
& Guerrero Rubén (1986) \\
& Lope Blanch (1996) \\
\hline Situacionales & Keller (1974) \\
& Kemper (1982) \\
& Uber (1999) \\
& Rojas (2014) \\
& Pérez (2018) \\
\hline Diacrónicos & Acevedo (1997) \\
& Company (1997) \\
\hline Sociolingüisticos & Lastra (1972) \\
& Kim Lee (1989 y 2007) \\
& Schwenter (1993) \\
& Reid y Comajoan (2005) \\
& Pejušković (2013) \\
\hline
\end{tabular}

Los estudios dialectológicos (Guerrero Rubín, 1985 y Guerrero Rubén, 1986) se basan en los resultados del Atlas Lingǘstico de México (Lope Blanch, 1996). Estas investigaciones se constriñen a una serie limitada de relaciones entre hablante/oyente, por lo general en el núcleo familiar (padre/hijo, esposo/esposa) y señalan el pronombre y la forma léxica que usan los individuos de una región en estas situaciones. Estos datos no permiten reconstruir 
el sistema global de tratamientos de una comunidad: "[...] no todos estos estudios permiten llegar a generalizaciones, pues su información es limitada con respecto a las relaciones interpersonales documentadas" (Vázquez y Orozco, 2010, p. 255). Por su parte, los estudios situacionales indagan por el tratamiento en contextos bien delimitados desde diferentes perspectivas: "Las disciplinas desde que éstas se llevan a cabo, de hecho, no siempre son lingǘsticas" (Vázquez y Orozco, 2010, p. 259); las relaciones de compadrazgo (Kemper, 1982), contextos laborales/comerciales (Uber, 1999), la alternancia pronominal en niños pequeños y sus cuidadores (Rojas, 2014) y los tratamientos en series televisivas (Pérez, 2018) son algunas de las situaciones que se examinan. Los estudios diacrónicos (Acevedo, 1997; Company Company, 1997) explican la pérdida de vosotros durante la época de la colonia desde diferentes puntos de vista.

Finalmente, los estudios de corte sociolingüístico (Lastra, 1972; Kim, 1989 y 2007; Schwenter, 1993; Reid y Comajoan, 2005; Pejušković, 2013) se caracterizan por utilizar cuestionarios como herramientas de elicitación; a diferencia de los estudios dialectológicos y situacionales, las investigaciones de este rubro tienen un espectro mucho más amplio, razón por la cual incluyen una gran cantidad de situaciones comunicativas y seleccionan a sus colaboradores según rasgos sociales como la edad, el sexo, la clase social y el nivel educativo. Los objetivos también difieren. Por un lado, se encuentran los estudios que tienen como fin describir y explicar el sistema de trato de la Ciudad de México (Lastra, 1972 y Kim, 1989); por otro lado, están las investigaciones que comparan los tratamientos de dos comunidades de habla dialectal y geográficamente diferentes (México y España) (Schwenter, 1993; Reid y Comajoan, 2005; Pejušković, 2013). Las diferencias también se observan en el tipo de muestra que seleccionan; aquellos estudios centrados en la capital mexicana obtienen datos únicamente de hablantes nacidos en la ciudad y son exhaustivos en que sus datos reflejen información social amplia, por lo tanto, incluyen la edad, el nivel educativo, el sexo y la clase social como factores de recolección y análisis. Por su parte, los estudios comparativos suelen concentrarse en un nivel educativo o en una clase social y agrupan bajo la etiqueta 'mexicanos' a sujetos provenientes de diferentes lugares de la República (Reid y Comajoan, 2005 y Pejušković, 2013): ${ }^{10 ~ “[. . .] ~ e s t o ~ n o ~ i m p l i c a ~ q u e ~ l o s ~}$ datos en todos los casos sean propios de estas ciudades, pues Schwenter, Reid Comajoan

\footnotetext{
10 No queda claro si los colaboradores del estudio de Schwenter son capitalinos o si el autor incluye a migrantes internos que habitan en la capital: "Los mexicanos encuestados residen actualmente en la ciudad de México" (Schwenter, 1993, p. 133).
} 
10 - Cepeda Ruiz, C. Y.

[...] no seleccionaron a sus informantes a partir de su origen" (Vázquez y Orozco, 2010, p. 258).

Grosso modo, los resultados de estas investigaciones señalan, por un lado, que el ustedeo es utilizado por adultos (Lastra, 1972; Kim Lee, 1989; Schwenter, 1993 y Pejušković, 2013) y en relaciones asimétricas entre jefes y subordinados y al dirigirse a desconocidos (Lastra, 1972 y Kim Lee, 1989). Por otro lado, el tuteo es frecuente entre jóvenes y personas de la misma edad (Lastra, 1972; Kim Lee, 1989; Schwenter, 1993 y Pejušković, 2013), sujetos pertenecientes a la clase alta (Lastra, 1972) y del nivel educativo superior (Kim Lee, 1989).

Especial atención merecen los hallazgos de Lastra (1972) y Kim Lee (1989). Ambos estudios utilizan cuestionarios sociolingüísticos y sus muestras están conformadas por capitalinos de varias generaciones, así como migrantes que han vivido en la ciudad desde pequeños (Kim Lee, 1989, p. 28). ${ }^{11}$

En su investigación, Lastra encuentra que tú predomina en relaciones familiares, en tanto que usted es más frecuente con individuos ajenos al núcleo familiar, especialmente con desconocidos; sin embargo, la autora señala que tú empieza a ocupar estos espacios y que dicho cambio es liderado por los jóvenes de la época:

Podemos decir que el tratamiento asimétrico va disminuyendo en la ciudad de México. El uso recíproco de tú va en aumento, sobre todo dentro de la familia, pero también fuera de ella, ya que se emplea inclusive para dirigirse a maestros y sacerdotes. Asimismo, el uso recíproco del tú está sustituyendo al usted, como en el caso de las conversaciones entre compradores y vendedores (Lastra, 1972, p. 215).

Por otro lado, los datos de Kim Lee compaginan bastante bien con los hallados por Lastra (1972) y difieren tan solo en algunos aspectos. El autor encuentra casi dos décadas después del trabajo de Lastra un ligero predominio de usted, esto se deduce de los resultados que presenta para las tres variables analizadas — sexo, edad y nivel educativo-:

[...] Como vimos en el análisis por sexo, usted predomina sobre tú en cada uno de los tres grupos generacionales. [...] Los tres grupos socioculturales usan más usted que tú en la totalidad de las situaciones estudiadas (Kim Lee, 1989, pp. 44, 58; subrayado en el original).

Finalmente, si bien Kim Lee concuerda con Lastra en que los jóvenes manejan pautas más tuteantes que los demás grupos generacionales, el autor considera que los hablantes más jóvenes no son exclusivamente tuteantes:

${ }^{11}$ La diferencia sustancial en la metodología de ambos estudios radica en que Lastra incluye la clase social como factor de análisis, en tanto que Kim Lee selecciona el nivel educativo. 
Como hemos observado hasta ahora, es cierto que los jóvenes tutean más que los adultos y ancianos en muchos actos de habla con personas con las que no sólo no han intimado previamente, sino que además no conocen ni han establecido con ellas un contacto personal. Sin embargo, a mi modo de ver, tú se emplea menos de lo que podría creerse, de conformidad con los resultados de mi encuesta, esto se comprueba por el hecho de que los jóvenes no prefieren emplear tú sino usted en algunos actos de habla con interlocutores jóvenes (Kim Lee, 1989, p. 56; subrayado en el original).

\section{Metodología}

\subsection{La muestra}

Los resultados de este estudio provienen de 48 cuestionarios sociolingüísticos aplicados a lo largo del año 2016 en la Ciudad de México. ${ }^{12}$ Los datos se recogieron mediante un muestreo no probabilístico por cuotas $^{13}$ según el sexo, edad, origen y nivel educativo de los participantes. ${ }^{14}$ Las cuotas de nuestra investigación no son exactas (hay más sujetos de nivel educativo alto y medio que del bajo, por ejemplo), pero sí aproximadas (en el caso del sexo). Las diferencias numéricas al interior de cada cuota se relacionan con la técnica 'bola de nieve' que utilizamos para recoger datos, estrategia que permitió trabajar con sujetos que integran grupos pequeños o micro-redes de estudiantes, familias bilingües, compañeros de oficina, entre otros.

La muestra está integrada por 27 mujeres y 21 hombres que pueden ser clasificados en cuatro generaciones: 11-24 años (14 sujetos), 25-34 (16), 35-54 (7) y +55 años (11); tres grupos según el nivel de escolaridad de los participantes: bajo — sin estudios/primaria trunca- (6 individuos), medio —secundaria/preparatoria- (20) y alto - estudios técnicos/universitarios - (22); dos grupos de origen: 33 personas nacidas en la Ciudad de México o 'capitalinos' y 15 migrantes internos que han vivido más de diez años en la capital y que provienen del Estado de México, Chiapas, Guerrero, Oaxaca, Puebla y Querétaro.

\footnotetext{
${ }^{12}$ La investigación general incluye resultados de 52 cuestionarios sociolingüísticos, además de datos extraídos de otras fuentes como corpus orales de la Ciudad de México.

13 Proceso que consiste en: "[...] dividir la población en subpoblaciones y en procurar que se atienda a informantes de todas ellas" (Moreno Fernández, 1990, p. 88).

${ }^{14} \mathrm{La}$ recolección de los datos, así como su análisis tuvo en cuenta los lineamientos propuestos en los materiales orales del Corpus Sociolingüístico de la Ciudad de México (Lastra \& Martín Butragueño, 2011, 2012 y 2015).
} 
3.1.1 $\operatorname{Sex} 0^{15}$

Los trabajos sociolingüísticos que incluyen la variable sexo suelen señalar que su incidencia en el fenómeno de estudio no es significativa y que, en el mejor de los casos, esta significatividad depende de la comunión con otras variables: "El sexo puede mostrarse, por tanto, más como un factor de segundo orden, como algo que suele subordinarse a dimensiones sociales diferentes y con mayor poder de determinación” (Moreno Fernández, 2009, p. 41). En el caso de los estudios sobre tratamientos del español en los que destaca esta variable se aprecian dos esquemas: uno, cuando los hablantes asumen patrones distintivos dependiendo de su sexo; y dos, cuando una forma (tú/vos) obtiene una marca negativa en la comunidad debido a su asociación con el sexo del hablante (véanse como ejemplos de esta marcación negativa: Carricaburo, 1997; Castro, 2001; Bartens, 2003; Moser, 2010a y 2010b; Chavarría, 2013 y Cepeda, 2014).

\subsubsection{Edad}

En numerosas investigaciones sobre tratamientos en el español la edad asume un papel mucho más relevante que otros factores sociales como el nivel educativo, el sexo y la clase social (Blas Arroyo, 2004, p. 302; Hummel, Kluge y Vázquez, 2010; Bertolotti, 2015). Por esta razón, a partir de la edad de los colaboradores llevamos a cabo un estudio en tiempo aparente (Labov, 1996, p. 99) que contrasta el comportamiento lingǘ́stico de cuatro generaciones que habitan la Ciudad de México; además, realizamos un estudio en tiempo real de corte cualitativo/cuantitativo (Labov, 1996, p. 135) que nos permite comparar los resultados actuales con los de estudios previos y observar el avance, retroceso o estabilidad de las formas pronominales en la capital mexicana. ${ }^{16}$ Como ya se ha señalado, los encuestados cuentan con edades que van desde los 11 hasta los 69 años y han sido agrupados en cuatro generaciones más o menos similares entre sí (los más jóvenes de la muestra: 11 a 24 años, los que se encuentran en la franja entre los 25 y los 34 años, los

\footnotetext{
15 Omitimos en estas páginas la discusión sobre las etiquetas 'género' y 'sexo', así como el interés cada vez más frecuente por la inclusión de nuevas categorías como 'identidad' y 'expresión de género'. Asumimos el sexo como una categoría biológica que divide a los sujetos en dos grupos: hombre y mujer y consideramos, tal como lo hace Blas Arroyo (2004, p.163) que su uso permite incluir de manera amplia factores sociales y culturales además de los biológicos.

16 Las comparaciones que hacemos, sin embargo, deben tener presente las diferencias inherentes a los materiales con los que contamos, los instrumentos con los que fueron recabados los datos (cuestionarios y entrevistas semidirigidas) y las características particulares de las muestras (dos, tres y cuatro categorías de edad, por ejemplo). En ese sentido, los resultados de nuestro análisis no se presentan como una interpretación inequívoca e irrefutable, sino como una posibilidad de integrar diferentes materiales para llevar a cabo nuevas observaciones.
} 
adultos entre los 35 y 54 años y los mayores de 55 años). ${ }^{17}$ Además de la edad absoluta de los colaboradores (dato que poseemos en todos los casos), en este estudio tomamos en cuenta la edad relativa del interlocutor e indagamos por el trato que usa el encuestado con personas mayores, menores o de la misma edad.

\subsubsection{Nivel educativo}

Trabajar con este factor es indudablemente menos controvertido que implementar un análisis a partir del concepto de clase social. Por una parte, los resultados de varios estudios indican que se relaciona con fenómenos de variación lingüística: "De hecho, la sociolingüística ha comprobado que este factor determina aisladamente numerosos hechos de variación, sin depender ni interaccionar con otras variables sociales" (Blas Arroyo, 2004, p. 228), véase por ejemplo los estudios de Kim Lee (1989), Bartens (2003), Cepeda (2014). Por otra parte, los parámetros que establecen las categorías del nivel de estudios — bajo, medio, alto - no dependen del investigador, sino de entidades gubernamentales (a diferencia de la clase social en la que el investigador suele crear los parámetros y escalas de análisis). Sin embargo, cabe señalar que el nivel educativo, al igual que la clase social, posee problemas metodológicos relacionados, uno, con las diferencias inherentes al sistema académico de cada comunidad, lo que dificulta las comparaciones a gran escala (por ejemplo, en Colombia la primaria comprende cinco grados, en tanto que en México se consideran seis); y dos, con los cortes y años de escolaridad que se incluyen para cada nivel (bajo, medio, alto), decisión que corre por cuenta del investigador.

\subsection{El instrumento}

La herramienta de recolección de datos de esta investigación es un cuestionario sociolingüístico adaptado de Cepeda (2014). ${ }^{18} \mathrm{El}$ instrumento posee tres cuadernillos: (1) información socioeconómica del colaborador, (2) formas pronominales y (3) fórmulas nominales. $^{19}$

\footnotetext{
17 "La división de grupos puede buscar, bien la agrupación de los informantes en categorías de dimensiones equivalente, marcando un límite más o menos objetivo cada cierto número de años (por ejemplo, cada 15 años: de 20 a 35, de 36 a 50, de 51 a 65), bien la agrupación en una misma categoría de los informantes que estén viviendo unas circunstancias vitales similares, sabiendo que éstas pueden variar de una comunidad a otra" (Moreno Fernández, 2009, p. 51).

18 Basado a su vez en el presentado por los integrantes del grupo PRESEEA Bogotá (Molina, 2002).

19 Tema del que no me ocuparé en estas líneas.
} 
El segundo cuadernillo, el que nos interesa en este estudio, se divide en cinco secciones: (1) Familia nuclear, (2) Familia extendida, (3) Trabajo/escuela, (4) Otros: Profesiones, desconocidos y (5) Percepción y valoración de los tratamientos. En las primeras cuatro secciones formulamos preguntas cerradas con tres opciones de respuesta —tú, usted y vos $-{ }^{20}$ Realizamos en estos apartados dos tipos de preguntas con el fin de apreciar la direccionalidad del tratamiento (Jaramillo, 1990, p. 16): — forma dirigida- o tratamiento que utiliza el colaborador en una situación determinada y — forma recibida-o trato que recibe el encuestado de su (inter)locutor. Por su parte, la sección cinco está conformada por preguntas abiertas sobre la valoración positiva o negativa que tienen los hablantes de los tratamientos pronominales. Los encuestados respondieron solo aquellas preguntas que se relacionan con su cotidianidad y dejaron en blanco situaciones en las que, por ejemplo, no recuerdan la forma que usan con su oyente o no existe el referente (padre, abuelo, etc.). ${ }^{21}$ de allí una diferencia entre los datos obtenidos para la forma dirigida —-1914 casos - y la forma recibida - 1817 respuestas-.

El cuestionario nos permitió recabar información de manera eficiente; pudimos evitar las generalizaciones, siendo exhaustivos en el número de interacciones que indagamos; la aplicación pudo hacerse en diferentes sesiones con lo que evitamos el cansancio de los encuestados; además, los resultados que obtenemos mediante este instrumento podrán ser cotejados, replicados o refutados en futuros estudios (García Córdoba, 2002; García Muñoz, 2003 e INEGI, 2013). ${ }^{22}$

En cuanto a los resultados, ofrecemos información de corte cuantitativo y cualitativo. Los datos correspondientes a las cuatro primeras secciones del cuestionario (preguntas cerradas sobre uso) fueron codificados a partir de los rasgos sociales sexo, edad y nivel educativo y analizados para brindar información descriptiva (frecuencias relativas y absolutas), además, incluimos un análisis de chi cuadrada $\left(\mathrm{X}^{2}\right)$ que tiene por objeto verificar la significatividad de cada una de las variables sociales en la selección de tú y usted. Finalmente, los resultados cuantitativos se complementan con datos cualitativos que se desprenden de la última sección del cuestionario (preguntas abiertas sobre percepción).

\footnotetext{
${ }^{20}$ Incluimos el pronombre vos además de tú y usted debido a que contamos con migrantes posiblemente voseantes en la muestra.

21 También se motivó a los participantes a señalar una segunda opción en caso de que existiera. Aunque esta última no se codificó cuantitativamente, sí se tuvo en cuenta al hacer un análisis cualitativo.

22 Una crítica mucho más detallada sobre las ventajas y desventajas de estos instrumentos puede encontrarse en Paredes (2010) y Orozco (2010).
} 


\section{Análisis y resultados}

\subsection{Resultados generales}

En la tabla 2 presentamos los resultados generales de este estudio (3731 datos). Para ello se tiene en cuenta la forma pronominal reportada por los encuestados, así como los dos tipos de preguntas realizadas forma dirigida y forma recibida.

\section{Tabla 2}

Uso general de las formas de tratamiento dirigidas y recibidas en el español de la Ciudad de México

\begin{tabular}{|l|c|c|c|}
\hline Tipo de respuesta & Tú & Usted & Total \\
\hline Forma dirigida & $1065 / 55.6 \%$ & $849 / 44.4 \%$ & $1914 / 100.0 \%$ \\
\hline Forma recibida & $1170 / 64.4 \%$ & $647 / 35.6 \%$ & $1817 / 100.0 \%$ \\
\hline
\end{tabular}

Los datos de la tabla 2 esbozan un sistema diádico en el que el pronombre tú suele codificar, dependiendo del contexto particular, cercanía, confianza, intimidad, informalidad (1a) $;^{23}$ en tanto que $u s t e d$ se usa en situaciones de distancia, formalidad y respeto (1b):

1. ¿Qué prefiere, tutear, ustedear, vosear?, ¿por qué?

a. Tutear, porque creo que da más confianza (Leonardo_h1ax). ${ }^{24}$

b. Ustedear, porque es menos problemático y más respetuoso (Carmen_m2cx).

Por un lado, los resultados agrupados bajo la etiqueta forma dirigida indican que tú es más utilizado que usted (55.6\% frente a 44.4\%); sin embargo, a pesar de que los 48 encuestados, de manera global, son más tuteantes, la diferencia entre ambos tratamientos no es abismal (rango de 11.2\%) y, de hecho, la frecuencia relativa de usted es bastante alta, lo que significa que esta forma posee contextos de uso bastante arraigados en la actualidad. Los datos son interesantes porque se ubican entre los obtenidos por Lastra (1972, p. 215), muestra en la que la autora parece encontrar un uso de tú mayoritario, y los de Kim Lee (1989, p. 33), quien observa un ligero predominio de usted. En ese sentido, los resultados de este estudio y la comparación con material previo dibujan un panorama en el que tú aumentó su

${ }^{23}$ Además, es habitual encontrar en el habla de la Ciudad de México el uso de tú en situaciones de enojo: * ¿Existe alguna situación en la que cambie la forma de tratamiento que usa cotidianamente, cuál, con quién? - Cambio el usted por el tú en situaciones de conflicto (Pedro_h4cx).

${ }^{24} \mathrm{El}$ código está compuesto por un seudónimo y las siglas correspondientes a los factores sociales sexo (h: hombre/m: mujer), edad (1: 11 a 24 años/ 2: 25 a 34 años/ 3: 35 a 54 años/ 4: más de 55 años), nivel educativo (b: bajo/c: medio/ a: alto) y origen (x: defeño/ i: migrante). 
16 - Cepeda Ruiz, C. Y.

frecuencia desde los años ochenta (Kim Lee, 1989), es decir, son reflejo de un aparente cambio lingüistico ${ }^{25}$ no obstante, los datos de la presente investigación son suficientes para señalar la generalización de tú y mucho menos para afirmar que se trate de la forma no marcada del español hablado en la capital mexicana.

Por otro lado, los resultados consignados en la tabla 2 bajo la etiqueta forma recibida reflejan el mismo patrón observado como forma dirigida, es decir, tú es más frecuente que usted. Adicionalmente, esta información indica que los colaboradores consideran que reciben tú como tratamiento más de lo que ellos tutean a sus interlocutores $(64.4 \%$ contra 55.6\%). El rango entre estos dos tipos de preguntas es de $8.8 \%$ y puede relacionarse con contextos e interacciones típicamente asimétricas en las que el locutor da $V$ y recibe $T$. Ejemplifican este tipo de situaciones las interacciones familiares de sujetos del nivel de estudios bajo y medio con figuras de autoridad, contextos en los que prima la semántica del PODER: con padres, abuelos y suegros emplean usted (2), en tanto que, con nietos, hijos, sobrinos reportan tú(3):

2. ¿A qué persona prefiere tratar de usted?

En general a los abuelos y suegros (Miguel_h1ci).

3. ¿A quién tutea con facilidad?, ¿por qué?

Amigos, sobrinos, hijos y nietos (Leonor_m3bi).

\subsection{Sexo}

Ahora bien, en la tabla 3 se presentan los resultados de la variable independiente sexo del informante (locutor). El lector puede apreciar que las diferencias entre hombres y mujeres son mínimas en cuanto a la forma dirigida y que en el caso de la forma recibida aumentan ligeramente (rango 10.5\%). Tú es más frecuente cuando el informante es hombre, ya sea que lo use o lo reciba, en tanto que usted es ligeramente superior cuando el encuestado es mujer:

\footnotetext{
${ }^{25}$ Recordemos que los resultados que comparamos en este estudio provienen de diferentes instrumentos, además, las muestra no son $100 \%$ equiparables y las situaciones comunicativas no son equilibradas; por lo tanto, las conclusiones que se derivan de este cotejo no pretenden ser incuestionables. 


\section{Tabla 3}

Formas pronominales de tratamiento dirigidas y recibidas en el español de la Ciudad de México, variable 'sexo del informante (locutor)' 26

\begin{tabular}{|c|c|c|c|c|c|c|}
\hline \multirow[b]{2}{*}{ Sexo } & \multicolumn{3}{|c|}{ FORMAS DIRIGIDAS } & \multicolumn{3}{|c|}{ FORMAS RECIBIDAS } \\
\hline & $T \dot{u}$ & Usted & Total & $T \dot{u}$ & Usted & Total \\
\hline Hombre & $\begin{array}{l}479 / \\
57.6 \%\end{array}$ & $\begin{array}{l}353 / \\
42.4 \%\end{array}$ & $\begin{array}{r}832 / \\
43.5 \% \\
\end{array}$ & $\begin{array}{c}558 / \\
70.3 \%\end{array}$ & $\begin{array}{l}236 / \\
29.7 \%\end{array}$ & $\begin{array}{r}794 / \\
43.7 \% \\
\end{array}$ \\
\hline Mujer & $\begin{array}{l}586 / \\
54.2 \%\end{array}$ & $\begin{array}{l}496 / \\
45.8 \%\end{array}$ & $\begin{array}{l}1082 / \\
56.5 \%\end{array}$ & $\begin{array}{l}612 / \\
59.8 \%\end{array}$ & $\begin{array}{l}411 / \\
40.2 \%\end{array}$ & $\begin{array}{l}1023 / \\
56.3 \%\end{array}$ \\
\hline Total & $\begin{array}{l}1056 / \\
55.6 \%\end{array}$ & $\begin{array}{c}849 / \\
44.4 \%\end{array}$ & $\begin{array}{c}1914 / \\
100.0 \%\end{array}$ & $\begin{array}{l}1170 / \\
64.4 \%\end{array}$ & $\begin{array}{c}647 / \\
35.6 \%\end{array}$ & $\begin{array}{c}1817 / \\
100.0 \%\end{array}$ \\
\hline
\end{tabular}

La pequeña diferencia en el trato usado/recibido por los hombres y las mujeres de la muestra se relaciona con el interés de ellos por entablar relaciones más íntimas con sus (inter)locutores (4a). Las mujeres, por su parte, señalan en repetidas ocasiones su necesidad por asentar límites y barreras físicas y psicológicas entre ellas y los sujetos de su entorno (Tannen, 1990, p. 89), especialmente si no hay lazos que los unan o si la relación y el contacto entre los hablantes es mínimo (4b). Las mujeres, además, asumen una postura más ustedeante que los hombres, debido a que consideran que este tratamiento es más respetuoso, elegante y formal que tú: "una serie de estudios sociolingüísticos han llegado a la conclusión de que las mujeres tienden a usar las variantes ligadas a un estatus alto más frecuentemente que los hombres" (Romaine, 1996, p. 100).

4. ¿Qué prefiere, tutear, ustedear, vosear?, ¿por qué?

a. Tutear porque es más cómodo y de más confianza (Juan_h4ax).

b. Ustedear, sobre todo cuando no conozco bien a la persona y no me interesa tener una relación estrecha con ella (Martha_m4ai).

Ahora bien, en la tabla 4 se presenta la combinación de los factores sexo del informante y sexo del (inter)locutor. Allí se aprecia que como forma dirigida el tuteo es ligeramente más usado en interacciones en las que intervienen sujetos del mismo sexo, ya sean hombres (58.8\%) o mujeres (55.8\%), patrón que recibe el nombre de solidaridad intrasexual (Carricaburo, 1997, p. 22); en tanto que usted aumenta su frecuencia sutilmente en contextos en los que las diferencias entre los hablantes, debido a su sexo, se hacen evidentes. En cuanto a la forma

${ }^{26}$ Forma dirigida: $N=1914, \mathrm{X}^{2}=2.25, p=0.13>0.05$, acepta $\mathrm{H}_{0}$. Forma recibida: $N$ : $1817, \mathrm{X}^{2}=21.3, p=0.00001$ $<0.05$, rechaza $\mathrm{H}_{0}$. 
18 - Cepeda Ruiz, C. Y.

recibida, los resultados confirman el esquema visto en la tabla 3, es decir, los informantes varones consideran que son más tuteados por hombres $(74.1 \%)$, mientras que las colaboradoras señalan que son más ustedeadas por los hombres (43.7\%) de su entorno.

Tabla 4

Formas pronominales de tratamiento dirigidas y recibidas en el español de la Ciudad de México, variables 'sexo del informante' y 'sexo del (inter)locutor ${ }^{27}$

\begin{tabular}{|c|c|c|c|c|c|c|}
\hline \multirow[b]{2}{*}{ Sexo H/O } & \multicolumn{3}{|c|}{ FORMAS DIRIGIDAS } & \multicolumn{3}{|c|}{ FORMAS RECIBIDAS } \\
\hline & $T \dot{u}$ & Usted & Total & $T \dot{u}$ & Usted & Total \\
\hline $\begin{array}{l}\text { Hombre } \\
\text { hombre }\end{array}$ & $\begin{array}{l}114 / \\
58.8 \% \\
\end{array}$ & $80 / 41.2 \%$ & $\begin{array}{c}194 / \\
100.0 \% \\
\end{array}$ & $\begin{array}{l}137 / \\
74.1 \% \\
\end{array}$ & $48 / 25.9^{\circ}$ & $\begin{array}{c}185 / \\
100.0 \% \\
\end{array}$ \\
\hline $\begin{array}{l}\text { Hombre } \\
\text { mujer }\end{array}$ & $\begin{array}{l}116 / \\
55.8 \%\end{array}$ & $92 / 44.2 \%$ & $\begin{array}{c}208 / \\
100.0 \%\end{array}$ & $\begin{array}{l}153 / \\
71.8 \%\end{array}$ & $60 / 28.5 \%$ & $\begin{array}{c}213 / \\
100.0 \%\end{array}$ \\
\hline Mujer a mujer & $\begin{array}{l}152 / \\
60.3 \%\end{array}$ & $\begin{array}{l}100 / \\
39.7 \%\end{array}$ & $\begin{array}{c}252 / \\
100.0 \%\end{array}$ & $\begin{array}{l}138 / \\
57.3 \%\end{array}$ & $\begin{array}{l}103 / \\
42.7 \%\end{array}$ & $\begin{array}{c}241 / \\
100.0 \%\end{array}$ \\
\hline $\begin{array}{l}\text { Mujer } \\
\text { hombre }\end{array}$ & $\begin{array}{c}149 / \\
55.8 \%\end{array}$ & $\begin{array}{l}118 / \\
44.2 \%\end{array}$ & $\begin{array}{c}267 / \\
100.0 \%\end{array}$ & $\begin{array}{l}138 / \\
56.3 \%\end{array}$ & $\begin{array}{l}107 / / \\
43.7 \%\end{array}$ & $\begin{array}{c}245 / \\
100.0 \%\end{array}$ \\
\hline
\end{tabular}

Tal como el lector puede apreciar, las diferencias entre tú y usted contenidas en la tabla 4 no son muy grandes, esto se replica a su vez en los pocos comentarios cualitativos que se encuentran en los cuestionarios relacionados con el sexo del (inter)locutor (5 y 6). La escasa referencia a esta variable resulta un indicio de la débil relación entre el factor sexo y los tratamientos pronominales tú y usted en la Ciudad de México.

5. ¿A quién no ustedearía o con quién considera que es complicado o inapropiado usar usted?, ¿por qué?

A los jóvenes varones, porque ellos te hablan más de tú (Eduardo_h3cx).

6. ¿A qué persona prefiere tratar de usted?

A las mujeres por poner una barrera (Eduardo_h3cx).

\subsection{Edad}

En la tabla 5 se observa la relación entre la variable dependiente y la edad del informante. A primera vista se aprecia que como forma dirigida tú predomina en las tres generaciones menores ( $7 \mathrm{a}, \mathrm{b}$ y c), en tanto que los mayores de 55 años reportan un uso más frecuente de usted (7d). Lo anterior es congruente con lo reportado en estudios previos sobre los tratamientos de otras variedades del español (Carricaburo, 1997; Hummel, Kluge

27 Forma dirigida: $N=921, \mathrm{X}^{2}=1.50639, p=0.68227>0.05$, acepta $\mathrm{H}_{0}$. Forma recibida: $N: 884, \mathrm{X}^{2}=24.8049$, $p=0.000017<0.05$, rechaza $\mathrm{H}_{0}$. 
y Vázquez, 2010; Quesada, 2013 y Bertolotti, 2015), así como en las investigaciones sobre la Ciudad de México (Lastra, 1972; Kim, 1989 y Schwenter, 1993), de hecho, esto último nos permite señalar la existencia de un patrón de estratificación por edad ${ }^{28}$ (Labov, 1996, p. 99): en la juventud los sujetos recurren preferentemente al tuteo y a medida que alcanzan su adultez asumen pautas más ustedeantes acordes con su nuevo rol en la sociedad y su visión de mundo.

\section{Tabla 5}

Formas pronominales de tratamiento dirigidas y recibidas en el español de la Ciudad de México, variable 'edad del informante (locutor) ${ }^{29}$

\begin{tabular}{|c|c|c|c|c|c|c|}
\hline \multirow{2}{*}{ Edad } & \multicolumn{3}{|c|}{ FORMAS DIRIGIDAS } & \multicolumn{3}{|c|}{ FORMAS RECIBIDAS } \\
\hline & $T \dot{u}$ & Usted & Total & $T \dot{u}$ & Usted & Total \\
\hline 11-24 años & $\begin{array}{l}335 / \\
62.3 \%\end{array}$ & $\begin{array}{l}203 / \\
37.7 \%\end{array}$ & $\begin{array}{l}538 / \\
28.1 \%\end{array}$ & $\begin{array}{l}410 / \\
79.5 \%\end{array}$ & $\begin{array}{c}110 / \\
20.5 \%\end{array}$ & $\begin{array}{l}516 / \\
28.4 \%\end{array}$ \\
\hline $\begin{array}{l}25-34 \\
\text { años }\end{array}$ & $\begin{array}{l}362 / \\
57.2 \%\end{array}$ & $\begin{array}{l}271 / \\
42.8 \%\end{array}$ & $\begin{array}{l}633 / \\
33.1 \%\end{array}$ & $\begin{array}{l}380 / \\
65.1 \%\end{array}$ & $\begin{array}{l}204 / \\
34.9 \%\end{array}$ & $\begin{array}{l}584 / \\
32.1 \%\end{array}$ \\
\hline 35-54 años & $\begin{array}{l}176 / \\
54.8 \% \\
\end{array}$ & $\begin{array}{r}145 / \\
45.2 \% \\
\end{array}$ & $\begin{array}{l}321 / \\
16.8 \% \\
\end{array}$ & $\begin{array}{l}161 / \\
53.0 \%\end{array}$ & $\begin{array}{r}143 / \\
47.0 \% \\
\end{array}$ & $\begin{array}{c}304 / \\
16.7 \%\end{array}$ \\
\hline+55 años & $\begin{array}{c}192 / \\
45.5 \%\end{array}$ & $\begin{array}{l}230 / \\
54.5 \%\end{array}$ & $\begin{array}{l}422 / \\
22.0 \%\end{array}$ & $\begin{array}{l}219 / \\
53.0 \%\end{array}$ & $\begin{array}{l}194 / \\
47.0 \%\end{array}$ & $\begin{array}{c}413 / \\
22.7 \%\end{array}$ \\
\hline Total & $\begin{array}{l}1056 / \\
55.6 \%\end{array}$ & $\begin{array}{l}849 / \\
44.4 \%\end{array}$ & $\begin{array}{c}1914 / \\
100.0 \%\end{array}$ & $\begin{array}{l}1170 / \\
64.4 \%\end{array}$ & $\begin{array}{l}647 / / \\
35.6 \%\end{array}$ & $\begin{array}{c}1817 / \\
100.0 \%\end{array}$ \\
\hline
\end{tabular}

7. ¿Qué prefiere, tutear, ustedear, vosear?, ¿por qué?

a. Tutear porque es más fácil para mí hablar así y estoy acostumbrado (Martín_h1cx).

b. Tutear, me siento menos incómodo, más en confianza (Santiago_h2cx).

c. Tutear porque se me hace más cómodo, a menos que pidan que no lo haga, depende de la relación (Margarita_m3cx).

d. Ustedear. Por educación y costumbre (Alma_m4ai).

Ahora bien, como forma recibida los resultados señalan que entre los 11 y los 34 (primera y segunda generación) los colaboradores consideran que son más tuteados por sus

28 "En la actualidad, la noción alude preferentemente a las diferencias en el lenguaje que son específicas de las diversas edades en la vida de los individuos y que contribuyen a singularizarlas desde un punto de vista" (Blas Arroyo, 2004, p. 193).

${ }^{29}$ Forma dirigida: $N=1914, \mathrm{X}^{2}=27.863, p=0.00001<0.05$, rechaza $\mathrm{H}_{0}$. Forma recibida: $N: 1838, \mathrm{X}^{2}=83.6483$, $p=0.00001<0.05$, rechaza $\mathrm{H}_{0}$. 
(inter)locutores, mientras que el ustedeo aumenta (sin ser el trato más frecuente) cuando el informante tiene 35 años en adelante (tercera y cuarta generación).

Varios comentarios deben hacerse con respecto a los datos de la tabla 5. Primero, la generación más ustedeante de la muestra, la cuarta, no usa usted exclusivamente, de hecho, los colaboradores de este grupo usan tú en un gran número de interacciones comunicativas de las propuestas en el cuestionario (45.5\%). Segundo, a pesar del prejuicio bastante extendido en la comunidad, la generación más joven no es plenamente tuteante. En efecto, tal como lo afirma Kim Lee, estos hablantes usan usted en varios contextos, incluso con sujetos de su misma edad:

[...] a mi modo de ver, tú se emplea menos de lo que podría creerse, de conformidad con los resultados de mi encuesta, esto se comprueba por el hecho de que los jóvenes no prefieren emplear tú sino usted en algunos actos de habla con interlocutores jóvenes. (Kim Lee, 1989, p. 56; subrayado en el original).

Tercero, los mayores de 55 años reportan recibir más tuteo del que dirigen a sus interlocutores, es decir, señalan un patrón no recíproco relacionado con su edad (8a). Este mismo esquema no simétrico es reportado por la primera generación, sujetos que consideran que sus (inter)locutores los tutean mucho más de lo que ellos usan esta forma (8b):

8. ¿Considera inapropiado que alguna(s) persona(s) lo tutee(n), quién(es)? ¿En qué situaciones le parece incorrecto que lo tuteen?

a. Me molesta de las empleadas de las tiendas o de algún servicio porque no son mis amigas y generalmente son muy jóvenes y yo les hablo de usted (Alma_m4ai).

b. Yo soy casi un niño así que si me tutean lo entiendo perfectamente, sí me gusta (Camilo_h1cx).

En cuanto a la edad del interlocutor, como forma dirigida las cuatro generaciones contempladas en este estudio reportan el uso de tú para tratar a personas de la misma edad o menores que ellos (9a y b):

Lo que se establece como el factor principal a la hora de elegir el trato con "figuras de respeto" es la percepción del destinatario como "igual" al hablante. Para esto influye el factor de la edad, es decir, cuando se trata de jóvenes mexicanos, si su destinatario se percibe como joven, hay un mayor grado de igualdad que cuando se trata de una persona mayor (Pejušković, 2013, p. 172). 
9. ¿A quién tutea con facilidad?, ¿por qué?

a. Gente de mi edad y menores (Alejandro_h2ai).

b. A los niños, a mi familia y a mis amigos (Alma_m4ai).

En tanto que usted se destina predominantemente al trato con personas mayores a los encuestados de los cuatro grupos de edad (10a y b), tal como reportan Lastra, 1972; Kim, 1989; Schwenter, 1993 y Pejušković, 2013:

10. ¿A qué persona prefiere tratar de usted?

a. Mayores, alguien con algún tipo de cargo, gente desconocido o que no es cercana (Hugo_h2ax).

b. Personas muy grandes, que las vea de 60 arriba, 50 arriba, 40 todavía tú (Jessica_m2ai).

Interesante resulta el trato dado por los mayores de 55 años a individuos de su misma edad, información contenida en el cuadro $63^{30}$ en este caso dos factores intervienen aportando valores opuestos que determinan la selección equilibrada de las formas tú y usted. Por un lado, la jerarquía superior inherente a las relaciones con sujetos mayores propicia el uso de usted; por otro lado, la situación solidaria que se establece entre miembros de un mismo grupo de edad favorece el uso de tú.

\section{Tabla 6}

Formas pronominales de tratamiento dirigidas y recibidas en el español de la Ciudad de México, variables 'edad del informante' $y$ 'edad del (inter)locutor ${ }^{31}$

\begin{tabular}{|l|c|c|c|c|c|c|}
\hline \multicolumn{8}{|c|}{ FORMAS DIRIGIDAS } \\
\hline \multirow{2}{*}{ Edad H/O } & MENOR QUE & \multicolumn{2}{|c|}{ IGUAL QUE } & \multicolumn{2}{c|}{ MAYOR QUE } \\
\cline { 2 - 8 } & Tú & Usted & Tú & Usted & Tú & Usted \\
\hline $11-24$ años & $26 / 92.9 \%$ & $2 / 7.1 \%$ & $28 / 100 \%$ & $0 / 0.0 \%$ & $1 / 3.6 \%$ & $27 / 96.4 \%$ \\
\hline $25-34$ años & $28 / 87.5 \%$ & $4 / 12.5 \%$ & $30 / 93.8 \%$ & $2 / 6.2 \%$ & $2 / 6.2 \%$ & $30 / 93.8 \%$ \\
\hline $35-54$ años & $14 / 87.5 \%$ & $2 / 12.5 \%$ & $11 / 68.8 \%$ & $5 / 31.2 \%$ & $2 / 12.5 \%$ & $14 / 87.5 \%$ \\
\hline+55 años & $18 / 75.0 \%$ & $6 / 25.0 \%$ & $12 / 50.0 \%$ & $12 / 50.0 \%$ & $2 / 8.3 \%$ & $20 / 91.7 \%$ \\
\hline \multicolumn{10}{|c|}{ FORMAS RECIBIDAS } \\
\hline
\end{tabular}

${ }^{30}$ La tabla 6 se basa en los datos extraídos de tres preguntas específicas que indagan sobre el tratamiento y la edad relativa del interlocutor.

${ }^{31}$ Forma dirigida: $N=298, \mathrm{X}^{2}=31.878, p=0.006689<0.05$, rechaza $\mathrm{H}_{0}$. Forma recibida: $N: 270, \mathrm{X}^{2}=47.514, p=$ $0.0000<0.05$, rechaza $\mathrm{H}_{0}$. 


\begin{tabular}{|l|c|c|c|c|c|c|}
\hline $11-24$ años & $22 / 84.6 \%$ & $4 / 15.4 \%$ & $24 / 92.3 \%$ & $2 / 7.7 \%$ & $16 / 61.5 \%$ & $10 / 38.5 \%$ \\
\hline $25-34$ años & $22 / 73.3 \%$ & $8 / 26.7 \%$ & $24 / 80.0 \%$ & $6 / 20.0 \%$ & $15 / 50.0 \%$ & $15 / 50.0 \%$ \\
\hline $35-54$ años & $4 / 28.6 \%$ & $10 / 71.4 \%$ & $3 / 21.4 \%$ & $11 / 78.6 \%$ & $2 / 14.3 \%$ & $12 / 85.7 \%$ \\
\hline+55 años & $12 / 60.0 \%$ & $8 / 40.0 \%$ & $12 / 60.0 \%$ & $8 / 40.0 \%$ & $7 / 35.0 \%$ & $13 / 65.0 \%$ \\
\hline
\end{tabular}

Como forma recibida la mayoría de los grupos reportan usted de parte de sujetos mayores y tú de individuos de su misma edad o menores (tal como sucede con la forma dirigida), sin embargo, algunas generaciones señalan diferencias interesantes. La tercera, por ejemplo, contrario a lo que pasa con los otros grupos, considera que es más ustedeada por sujetos menores (71.4\%) y de su misma edad (78.6\%); este comportamiento se relaciona, en parte, con el estado de transición que atraviesan los hablantes entre los 35 y 54 años, situación en la que la imagen exterior asumida por el colaborador y sus (inter)locutores determina el mayor uso de usted (11a y b):

11. ¿Considera inapropiado que alguna(s) persona(s) lo ustedee (n), quién(es)? ¿En qué situaciones le parece incorrecto que lo ustedeen?

a. No; ya se acostumbra uno, la primera vez que me dijeron me sentí viejo (Eduardo_h3cx).

b. Sí, solo porque me siento mayor (Ángeles_m3ax).

Otro cambio en la direccionalidad del tratamiento se observa entre la forma dirigida y la recibida por los sujetos entre los 11 y 34 años (primera y segunda generación) en intercambios comunicativos con sujetos mayores que ellos. Los dos grupos más jóvenes reportan usted como trato privilegiado al apelar a individuos mayores que ellos $(96.4 \%$ de la primera generación y 93.8\% de la segunda); en tanto que como forma recibida los jóvenes consideran ser más tuteados $(61.5 \%$ la primera generación y $50.0 \%$ la segunda). Este resultado no es extraño y es reflejo de una relación de poder que se caracteriza porque el sujeto que es visto como superior (el mayor) da $T$, en tanto que el menor usa $V$ (Brown y Gilman, 1960), esquema que ya hemos señalado en secciones previas y que, de cierta manera, se opone a expectativa general que considera el aumento del trato simétrico solidario (Brown y Gilman, 1960; Lastra, 1972; Wainerman, 1976; Carricaburo, 1997 y Fontanella de Weinberg, 1999; entre otros).

\subsection{Nivel educativo}


La variable nivel educativo tuvo en cuenta tan solo información respecto a la forma dirigida, esto se debe a que los colaboradores, excepto pocos comentarios de corte cualitativo (12), no señalaron la incidencia de este factor en la selección en el tratamiento pronominal.

12. ¿Existe alguna situación en la que cambie la forma de tratamiento que usa cotidianamente?, ¿cuál?, ¿con quién?

Cuando es una persona mayor o que sabe mucho (Miguel_h1ci).

Los resultados de la tabla 7 indican que los sujetos con estudios medios (preparatoria) y altos (técnicos y universitarios) usan con más frecuencia tú al dirigirse a sus interlocutores (59.6\% y $57.5 \%$, respectivamente), sin que este sea un uso generalizado; por su parte, los colaboradores de esta muestra que cuentan con nivel de estudios básico (primaria incompleta y sin estudios) prefieren usted como trato pronominal de segunda persona $(62.9 \%)$.

\section{Tabla 7}

Formas pronominales de tratamiento dirigidas en el español de la Ciudad de México, variable 'nivel educativo del informante (locutor) 32

\begin{tabular}{|l|c|c|c|}
\hline Nivel educativo & Tú & Usted & Total \\
\hline Bajo & $93 / 37.1 \%$ & $158 / 62.9 \%$ & $251 / 13.1 \%$ \\
\hline Medio & $455 / 59.6 \%$ & $309 / 40.4 \%$ & $764 / 39.9 \%$ \\
\hline Alto & $517 / 57.5 \%$ & $382 / 42.5 \%$ & $899 / 47.0 \%$ \\
\hline Total & $1056 / 55.6 \%$ & $849 / 44.4 \%$ & $1914 / 100.0 \%$ \\
\hline
\end{tabular}

Los resultados se asemejan al patrón reportado por Kim Lee en su estudio (1989), así como a los datos de otras variedades del español en las que se asocia el tuteo con el habla de sujetos con estudios medios y altos y el ustedeo con individuos con un bajo nivel de escolaridad (Carricaburo, 1997). No obstante, debe tenerse en cuenta que los datos contenidos en la tabla 7 reflejan el cruce de varias variables. Los sujetos del nivel bajo de estudios pertenecen, uno, a la cuarta generación, dos, a grupos migrantes que tienen dominio activo y pasivo de una lengua indígena (matlazinca, mixteco, zapoteco y náhuatl), es decir, colectivos altamente ustedeantes; en tanto que los informantes con estudios medios y altos son mayoritariamente capitalinos y pertenecen a los grupos de edad más jóvenes de la muestra, es decir, sectores promotores del tuteo.

\footnotetext{
${ }^{32}$ Forma dirigida: $N=1914, \mathrm{X}^{2}=41.1537, p=0.00001<0.05$, rechaza $\mathrm{H}_{0}$. 


\section{Conclusiones}

En este artículo analizamos las formas pronominales tú y usted en conjunción con los factores sexo, edad y nivel educativo. Los datos reflejan no solo el sistema de tratamientos utilizado en la Ciudad de México, sino las diferentes concepciones del entorno social de quienes participan en el estudio, así como la relevancia y jerarquización de los parámetros extralingüísticos.

Los datos indican que actualmente tú es el tratamiento más utilizado en la Ciudad de México (forma dirigida/recibida), sin embargo, su frecuencia no es abrumadora. Por su parte, usted se utiliza con carácter normativo en contextos en los que predomina la semántica del poder, con lo cual de dificulta la pronta expansión de tú. En consecuencia, nuestros resultados confirman parcialmente las tesis generales de Lastra (1972) y Kim Lee (1989). Por un lado, la frecuencia de tú es superior a la de usted, tal como afirma Lastra; por otro lado, usted no ha sido desplazado totalmente por tú, pues en situaciones específicas, según señala Kim Lee (1989), no hay una buena apreciación de este trato, incluso entre la población joven.

Más allá, consideramos prudente realizar futuras investigaciones que examinen el tuteo y ustedeo en situaciones comunicativas particulares (la familia, en contextos de compra/venta, etc.) para determinar en dichas situaciones un posible cambio lingüistico; la frecuencia general actual, comparada con los datos ligeramente ustedeantes de Kim Lee (1989), nos empuja a pensar que en efecto se trata de un cambio, sin embargo, la naturaleza de las muestras y de los datos nos impide negar que se trate de un efecto residual de la investigación.

Los resultados determinan que las variables sociales analizadas son cuantitativa y cualitativamente relevantes en la elección de las formas pronominales. Específicamente, el análisis de $\mathrm{X}^{2}$ considera significativas estadísticamente la edad del colaborador/ (inter)locutor, el nivel educativo del informante y el sexo del (inter)locutor, en tanto que descarta el sexo del locutor como factor significante en la selección del trato.

Si bien el sexo no es significativo estadísticamente, apreciamos en los resultados descriptivos y cualitativos un esquema relacionado con este factor: tú es más reportado por los varones de la muestra tanto como forma dirigida, como recibida, en tanto que las mujeres prefieren pautas ustedeantes (las diferencias porcentuales son mínimas). La afinidad de los hombres por el tuteo se refleja en comentarios cualitativos que señalan su interés por 
establecer relaciones íntimas con los sujetos de su entorno. Ellas, en cambio, están conscientes de su papel 'vulnerable' en la sociedad (Tannen, 1990, p. 127), especialmente en la mexicana y recurren al lenguaje, específicamente a las formas de tratamiento, como estrategia de defensa (Bosque, 2012, p. 3). Al examinar el trato dirigido en relación con el sexo del informante y de su (inter)locutor observamos que las personas del mismo sexo se tratan de tú como pauta solidaria (Carricaburo, 1997, p. 22), en tanto que en conversaciones entre hombres y mujeres la forma más frecuente es usted.

La edad resulta una de las variables más interesantes. Como forma dirigida se aprecia un patrón de estratificación por edad (Labov, 1996, p. 99): los jóvenes de la muestra actual, tal como sucede en los estudios previos (Lastra, 1972, pp. 214-215; Kim Lee, 1989, p. 48 y Schwenter, 1993, p. 136), prefieren el tuteo, los adultos, por su parte escogen en más ocasiones usted; sin embargo, el prejuicio asociado a la edad no es siempre cierto: los jóvenes no son exclusivamente tuteantes y los adultos no son plenamente ustedeantes. Como forma recibida observamos algunos cambios en la direccionalidad y frecuencia del tratamiento: los jóvenes de la primera generación, por ejemplo, los más tuteantes del estudio, reciben más tú del que dan, evidencia de un patrón asimétrico, un contexto en el que domina la semántica del poder. Adicionalmente, la edad del interlocutor refleja que los sujetos mayores son ustedeados por las cuatro generaciones, mientras que a los menores y a los de igual edad se les trata de tú; los individuos de la cuarta generación, particularmente, deciden entre la solidaridad y el poder cuando se dirigen a sus contemporáneos mayores de 55 años.

Por su parte, el nivel educativo reflejó el mismo patrón reportado por Kim Lee (1989, p. 58): tú es promovido por los sujetos con estudios medios y altos y usted por colaboradores de nivel bajo. Estos datos se relacionan con la variable edad, los sujetos que pertenecen al grupo de estudios bajo son mayores de 55 años (más ustedeantes), en tanto que los que conforman el nivel medio y alto son sujetos de la primera y segunda generación (más tuteantes).

Finalmente, queda por estudiar la relación del trato pronominal y variables pragmático-discursivas como el tipo de acto de habla, el tema, el estado anímico de los participantes del evento comunicativo, así como la interacción entre tratos pronominales y nominales.

\section{Referencias}


Bartens, Á. (marzo de 2003). Notas sobre el uso de las formas de tratamiento en el español colombiano actual, Pronoms de 2 personne et forms d'adresse dans les Langues d'Europe. Coloquio de Paris. Instituto Cervantes, Paris. Recuperado de https://cvc.cervantes.es/lengua/coloquio paris/ponencias/bartens.htm

Bertolotti, V. (2015). A mi de vos no me trata ni usted ni nadie. Sistemas e historia de las formas de tratamiento en la lengua española en América. Ciudad de México: Universidad Nacional autónoma de México/Universidad de la República Uruguay.

Blas Arroyo, J. L. (2004). Sociolingüistica del español. Desarrollos y perspectivas en el estudio de la lengua española en contexto social. Madrid: Cátedra.

Bosque, I. (2010). Sexismo lingüistico y visibilidad de la mujer. Madrid: Real Academia Española.

Brown, R. \& Gilman, A (1960). The pronouns of power and solidarity. En Sebeok T. (ed.), Style in Language (pp. 253-276. Cambridge: MIT Press.

Calderón, M. (2010). Los elementos nominales en el sistema de tratamientos del español de Andalucía durante la Restauración (1875-1931). En M. Hummel, B. Kluge \& M. E. Vázquez (eds.), Formas y fórmulas de tratamiento en el mundo bispánico (pp. 551-570). Ciudad de México: El Colegio de México/Karl Franzens Universität.

Carricaburo, N. (1997). Las fórmulas de tratamiento en el español actual. Madrid: Arco/Libros.

Castro, A. (2001). Los pronombres de tratamiento en el español de Honduras. Múnich: Lincom Europe.

Chavarría, C. (2013). El español de Guatemala: nivel morfosintáctico. En M. Á, Quesada (ed.), El español bablado en América Central: nivel morfosintáctico (pp. 65-140). Madrid/Frankfurt: Iberoamericana/Vervuert.

Cepeda, C. Y. (2014). Usted, tú, sumercé y vos: formas pronominales de tratamiento en el español de Bogotá (Colombia). (Tesis de maestría). Universidad Nacional Autónoma de México, Ciudad de México. Recuperado de http://132.248.9.195/ptd2014/octubre/513451939/Index.html

Company Company, C. (1994). El costo gramatical de las cortesías en español americano. Consecuencias sintácticas de la pérdida de vosotros. Anuario de letras, 35, 167-191.

Recuperado de http://www.concepcioncompany.com/1997/08/1997-el-costogramatical-de-las.html

Crystal, D. (2008). A Dictionary of Linguistics and Phonetics. 6th Ed. Maiden: Blackwell Publishing. https://doi.org/10.1002/9781444302776

Fontanella de Weinberg, M. B. (1999). Sistemas pronominales de tratamiento usados en el mundo hispánico. En I. Bosque \& V. Demonte (Dirs.), Gramática Descriptiva de la Lengua Española. Tomo 3 (pp. 1399-1426). Madrid: Espasa.

García Córdoba, F. (2002). El cuestionario. Recomendaciones metodológicas para el diseño de cuestionario, Ciudad de México: Limusa.

García Muñoz, T. (2003). El cuestionario como instrumento de investigación/evaluación, España: Centro Universitario Santa Ana. Recuperado de http://www.univsantana.com/sociologia/El Cuestionario.pdf

Guerrero Rubén, J. L. [sic; Rubín] (1986). Diferencias léxicas entre el habla culta y la popular en la vida social de la Ciudad de México, Actas del II Congreso Internacional sobre el español de América (pp. 531-538). Ciudad de México, Universidad Nacional Autónoma de México.

Guerrero Rubín, J. L. (1985). El léxico de la "vida social y diversiones" en la Ciudad de México. (Tesis de licenciatura), Universidad Nacional Autónoma de México, Ciudad de México. 
Hummel, M., Kluge, B., \& Vázquez, M. E. (eds.). (2010). Formas y fórmulas de tratamiento en el mundo bispánico. Ciudad de México: El Colegio de México/Karl Franzens Universität.

INEGI (Instituto Nacional de Estadística y Geografía) (2013). Clases medias en México. Boletin de Investigación 256/13. Recuperado de http://usmex2024.uscmediacurator.com/wp-content/uploads/2013/08/ClasesMedias-en-Mexico.pdf

Jaramillo, J. A. (1990). Domain constraints on the use of TÚ and USTED. En J. Bergen (ed.), Spanish in the United States: Sociolinguistics issues (pp. 14 -22). Washington: Georgetown University Press.

Kemper, R. V. (1982). The compadrazgo in urban Mexico. Anthropological Quarterly. 55 (1), 17-30. https://doi.org/10.2307/3317372

Kim Lee, U. S. (1989). El uso de tú y usted en el español de la Ciudad de México. (Tesis de maestría). Universidad Nacional Autónoma de México, Ciudad de México. Recuperado de http://132.248.9.195/pmig2018/0097749/Index.html

Kim Lee, U. S. (2007). Observaciones sobre el uso de las fórmulas de tratamiento de los jóvenes mexicanos. Iberoamericana. 9 (1), 159-177. Recuperado de http://www.iberoamerica.kr/data/file/9-1-07.pdf

Labov, W. (1996). Principios del cambio lingüistico. 1: Factores internos. Madrid: Gredos.

Lastra, Y. (1972). Los pronombres de tratamiento en la Ciudad de México. Anuario de Letras. 10, 213-217. Recuperado de https://revistas-filologicas.unam.mx/anuarioletras/index.php/al/article/view/277/276

Lastra, Y. \& Martín Butragueño, P. (Coords.). (2011). Corpus sociolingüístico de la ciudad de México. Vol. 1: Nivel alto. Ciudad de México: El Colegio de México. Recuperado de http://lef.colmex.mx/index.php/investigaciones/corpus-sociolingueistico-de-laciudad-de-mexico-cscm

Lastra, Y. \& Martín Butragueño, P. (Coords.). (2012). Corpus sociolingüistico de la ciudad de México. Vol. 2: Nivel medio. Ciudad de México: El Colegio de México. Recuperado de http://lef.colmex.mx/index.php/investigaciones/corpus-sociolingueistico-de-laciudad-de-mexico-cscm

Lastra, Y. \& Martín Butragueño, P. (Coords.). (2015). Corpus sociolingüistico de la ciudad de México. Vol. 2: Nivel bajo. Ciudad de México: El Colegio de México. Recuperado de http://lef.colmex.mx/index.php/investigaciones/corpus-sociolingueistico-de-laciudad-de-mexico-cscm

Lope Blanch, J. M. (Dir.). (1996). Atlas lingüistico de México. vol. 4. Morfosintaxis. Ciudad de México: El Colegio de México/Universidad Nacional Autónoma de México.

Molina, I. (2002). Cuestionario sobre las formas de tratamiento. Bogotá: PRESSEA.

Montes Giraldo, J. J. (1985). El español bogotano en 1983 muestra fonética y gramatical. THESAURUS. XL (2), 294-307. Recuperado de https://cvc.cervantes.es/lengua/thesaurus/pdf/40/TH $40 \quad 002 \quad 053 \quad 0 . p d f$

Moreno Fernández, F. (1990). Metodología sociolingüística. Madrid: Gredos S.A.

Moreno Fernández, F. (2009). Principios de sociolingüística y sociología del lenguaje. 4ta ed. Barcelona: Ariel S.A.

Moser, K. (2010a). Las formas de tratamiento verbales-pronominales en Guatemala, El Salvador, Panamá (y Costa Rica): hacia una nueva sistematización en la periferia centroamericana. En M. Hummel, B. Kluge \& M. E. Vázquez (eds.), Formas y fórmulas de tratamiento en el mundo bispánico (pp. 271-291). Ciudad de México: El Colegio de México/Karl Franzens Universität. 
Moser, K. (2010b). San José (Costa Rica): desde los dignificados pragmáticos del ustedeo en el registro coloquial actual hacia sus primeras manifestaciones en el valle central (siglo XVIII). En M. Hummel, B. Kluge \& M. E. Vázquez (eds.), Formas y fórmulas de tratamiento en el mundo bispánico (pp. 671-713). Ciudad de México: El Colegio de México/Karl Franzens Universität.

Oliveira, S. M (2010). La integración de la teoría y la metodología como desencadenante de un nuevo modelo de formas y fórmulas de tratamiento. En M. Hummel, B. Kluge \& M. E. Vázquez (eds.), Formas y fórmulas de tratamiento en el mundo bispánico (pp. 5777). Ciudad de México: El Colegio de México/Karl Franzens Universität.

Orozco, M. L. (2010). Estudio sociolingüístico de la cortesía en tratamientos y peticiones. Datos de Guadalajara. (Tesis de doctorado). El Colegio de México, Ciudad de México.

Paredes, Fl. (2010). ¿Es factible un cuestionario estándar para el estudio del tratamiento? La experiencia del proyecto PRESSEA en Madrid y Alcalá de Henares. En M. Hummel, B. Kluge \& M. E. Vázquez (eds.), Formas y fórmulas de tratamiento en el mundo hispánico (pp. 164-191). Ciudad de México: El Colegio de México/Karl Franzens Universität.

Pejušković, M. (2013). Tú y usted: dos pronombres de tratamiento en una comunidad de mexicanos residentes en Madrid. Lingüistica Mexicana. VII (2), 159-191. Recuperado de http://amla.org.mx/linguistica mexicana/Vol VII 2/2013070204a.pdf

Pérez, F. (mayo de 2018). Formas de tratamiento en el habla actual de la Ciudad de México. III Congreso Formas y Fórmulas de Tratamiento del Mundo Hispánico y Luso-Brasileño. Brasil, Universidad Federal de Santa Catarina.

Quesada, M. Á. (ed.). (2013). El español hablado en América Central: nivel morfosintáctico. Madrid: Iberoamericana/Vervuert.

Real Academia Española-Asociación de Academias de la Lengua (2009). Las formas de tratamiento. En Nueva Gramática de la Lengua Española. Tomo 1 (pp. 1250-1268). Madrid: Espasa.

Rojas, C. (2014). Afecto y control. Cambio T > V en el tratamiento a los niños pequeños. En P. Martín Butragueño \& L. Orozco (eds.), Argumentos cuantitativos y cualitativos en sociolingüística (701-734). Ciudad de México: El Colegio de México.

Romaine, S. (1996). El lenguaje en la sociedad. Una introducción a la sociolingüística. Barcelona: Ariel.

Schwenter, S (1993). Diferenciación dialectal por medio de pronombres: una comparación del uso de tú y usted en España y México. Nueva Revista de Filología Hispánica. 21, 127-149. Recuperado de http://nrfh.colmex.mx/index.php/nrfh/article/view/926 https://doi.org/10.24201/nrfh.v41i1.926

Vázquez, M. E., \& Orozco, M. L. (2010). "Formas de tratamiento del español de México". En M. Hummel, B. Kluge \& M. E. Vázquez (eds.), Formas y fórmulas de tratamiento en el mundo hispánico (pp. 248-269). Ciudad de México: El Colegio de México/Karl Franzens Universität.

Wainerman, C. (1976). Sociolingüistica de la forma pronominal. Ciudad de México: Trillas.

Cristal Yeseidy Cepeda Ruiz es candidata al grado de doctor en lingüística de la Universidad Nacional Autónoma de México (UNAM) (febrero de 2018). Maestra en 
Lingüística Hispánica con Mención Honorífica por la UNAM (2014). Becaria del Consejo Nacional de Ciencia y Tecnología de México (CONACyT) durante los estudios de maestría y doctorado (2012-2014 y 2015-2019). Reconocimiento del Comité Académico del Programa de Posgrado de la UNAM por la tesis "Usted, tú, sumercé y vos: formas pronominales de tratamiento en el español de Bogotá, Colombia” (2014). Escritora y ponente de varios estudios sobre las formas de tratamiento del español hablado en Bogotá y Ciudad de México. Miembro de AMLA (Asociación Mexicana de Lingüística Aplicada), ALFAL (Asociación de Lingüística y Filología de América Latina) y participante asiduo en los eventos organizados por el grupo de investigadores sobre formas de tratamiento del mundo hispánico y luso-brasileño.

Cristal Yeseidy Cepeda Ruiz is a candidate to the degree of doctor in linguistics by Universidad Nacional Autónoma de México (UNAM) (february 2018). Master in Hispanic Linguistics with Honorary Mention by UNAM (2014). Scholarship holder of the Consejo Nacional de Ciencia y Tecnología de México (CONACyT) during the master's and doctorate studies (2012-2014 and 2015-2019). Recognition by the Academic Committee of the Graduate Program of the UNAM for the thesis " Usted, tú, sumercé y vos: formas pronominales de tratamiento en el español de Bogotá, Colombia " (2014). Writer and speaker of several studies about the forms of treatment of spoken Spanish in Bogotá and Mexico City. Member of AMLA (Asociación Mexicana de Lingüística Aplicada), ALFAL (Asociación de Lingüística y Filología de América Latina) and regular participant in the events organized by the group of researchers on forms of adress in the Hispanic and Portuguese-Brazilian world. 